Pacific Journal of Mathematics

SOME FUNDAMENTAL PROPERTIES OF CONTINUOUS 


\title{
SOME FUNDAMENTAL PROPERTIES OF CONTINUOUS FUNCTIONS AND TOPOLOGICAL ENTROPY
}

\author{
ROMEO F. THOMAS
}

\begin{abstract}
The purpose of this paper is to clarify some properties and results related to continuous functions on compact spaces and topological entropy.
\end{abstract}

1. Definitions and Propositions. Note that in this paper we assume that the spaces are compact metric spaces unless otherwise stated.

If $\alpha, \beta$ are open covers of $X$ their join

$$
\alpha \vee \beta=\{A \cap B: A \in \alpha \text { and } B \in \beta\} \text {. }
$$

We define $H(\alpha)=\log N(\alpha)$, where $N(\alpha)$ is the number of sets in a finite subcover of $\alpha$ for $X$ with smallest cardinality. Note that $H(\alpha) \geq$ 0 and $H(\alpha \vee \beta) \leq H(\alpha)+H(\beta)[\mathbf{1 0}]$. Define

$$
\operatorname{diam}(\alpha)=\max \{\operatorname{diam}(U): U \in \alpha\} .
$$

Let $(X, \phi)$ denote a continuous real flow [i.e., $\phi: X \times R \rightarrow X$ continuous and $\phi(x, t+s)=\phi(\phi(x, t), s)]$ on a compact metric space $X$. The topological entropy of $\phi$ is denoted by $h(\phi)$ and defined to be $h(\phi)=h\left(\phi_{1}\right)$, where $\phi_{t}: X \rightarrow X$ is a homeomorphism defined by $\phi_{t}(x)=\phi(x, t)$.

We recall that the flows $(X, \phi)$ and $(Y, \varphi)$ are conjugate (topologically conjugate) if there is a homeomorphism $\gamma$ from $X$ onto $Y$ mapping orbits of $\phi$ onto orbits of $\varphi$ with preserved orientation. For more details see $[2,8,9]$.

Proposition 1.1 (cf. [5]). If $(X, \phi)$ and $(Y, \psi)$ are conjugate flows and they have no fixed points, then

$$
h(\phi)=\operatorname{ch}(\psi),
$$

where $c$ is a finite positive constant.

Let $T: X \rightarrow X$ be a homeomorphism and let $f: X \rightarrow R$ be any positive real valued continuous function. The suspension of $T$ under 
$f[2,8]$ is defined to be the flow $\phi_{f}$ on the space

$$
X_{f}=\bigcup_{0 \leq t \leq f x}\{(x, t):(x, f x) \sim(T x, 0)\}
$$

defined for small non-negative time by $\phi_{f_{s}}(x, t)=\phi_{f}(x, t+s)$ with $0 \leq t+s \leq f x$.

It is well known that the suspension flows $\left(X_{f}, \phi_{f}\right)$ and $\left(X_{g}, \phi_{g}\right)$ of $T: X \rightarrow X$ under $f$ and $g$ respectively are conjugate and a homeomorphism from $X_{f}$ onto $X_{g}$ that conjugates the flows is given by $(x, t) \rightarrow(x, \operatorname{tg}(x) / f(x))$.

Let $d_{1}$ and $d_{2}$ be metrics defined on $X$. These metrics are Lipschitzequivalent (L-equivalent) if there exist positive constants $c_{1}$ and $c_{2}$ such that

$$
c_{1} d_{1}(x, y) \leq d_{2}(x, y) \leq c_{2} d_{1}(x, y)
$$

for every $x, y \in X$. A map $f$ from a metric space $(X, d)$ into a metric space $(Y, \sigma)$ is Lipschitz (L-map) if there exists a positive constant $c$ such that

$$
\sigma(f x, f y) \leq c d(x, y)
$$

for every $x, y \in X$. A Lipschitz bijective map $f: X \rightarrow Y$ such that $f^{-1}$ is also Lipschitz will be called L-homeomorphism and denoted by $f: X \cong Y$. A metric space $(X, d)$ is $L$-embedded in a metric space $(Y, \sigma)$ if there exists an injective $L$-map $i: X \rightarrow Y$ such that $X \cong$ $i(X) \subseteq Y$.

It is obvious that any compact differentiable manifold $M$ with the Riemannian metric is $L$-embedded in the Euclidean space $R^{m}$ for some positive integer $m$.

2. Continuous functions. In this section we will introduce our basic proposition.

Propostion 2.1. Let $f: X \rightarrow R$ be a positive real valued continuous function on $X$ (compact metric space). Given $\varepsilon>0$, then for every positive integer $n$, there exists an open cover $\alpha_{n}$ of $X$ such that $\operatorname{diam}(f U) \leq \varepsilon / n$ for all $U$ in $\alpha_{n}$ and

$$
\lim _{n \rightarrow \infty} \frac{1}{n} H\left(\alpha_{n}\right)=0 .
$$

Proof. Let $\left(Y=Y_{f}, \phi=\phi_{f}\right)$ be the suspension flow of the identity map $I: X \rightarrow X$ under the given function $f$. Since $(Y, \phi)$ is conjugate to the suspension flow $\left(X_{1}, \phi_{1}\right)$ of $I: X \rightarrow X$ under the constant 1 and $h\left(\phi_{1}\right)=h(I)=0$, Proposition 1.1 implies that $h(\phi)=0$. Now given 
$n>0$, let $t_{n}=n \sup _{x \in X}(f x)$ and take $E_{n}$ to be $\left(t_{n}, \varepsilon / 2\right)$-spanning set of $X \times\{0\}$ with respect to $\phi$ and with minimum cardinality. For $e \in E_{n}$, let

$$
U_{e}=\left\{x \in X: d\left(\phi_{s} x, \phi_{s} e\right)<\varepsilon / 2 \text { for } 0 \leq s \leq t_{n}\right\} .
$$

Then $U_{e}$ is an open neighborhood of $e$. Suppose $\operatorname{diam}\left(f U_{e}\right)=\lambda_{e}$. Then $\operatorname{diam}\left(U_{e}\right)+m \cdot \lambda_{e} \leq \varepsilon$ for some $m \geq n$. Hence $\lambda_{e} \leq \varepsilon / m \leq \varepsilon / n$. Let $\alpha_{n}=\left\{U_{e}: e \in E_{n}\right\}$. It is clear that $\alpha_{n}$ is an open cover of $X$ and $\operatorname{card}\left(\alpha_{n}\right) \leq \operatorname{card}\left(E_{n}\right)$. Since

$$
\lim _{n \rightarrow \infty} \frac{1}{t_{n}} \log \operatorname{card}\left(E_{n}\right) \leq h(\phi)
$$

and $h(\phi)=0$, therefore

$$
\lim _{n \rightarrow \infty} \frac{1}{n} H\left(\alpha_{n}\right) \leq \lim _{n \rightarrow \infty}\left(\sup _{x \in X} f(x) / t_{n}\right) \log \operatorname{card}\left(E_{n}\right)=0,
$$

and the proof is finished.

Claim 1. Let $f: X \rightarrow R$ be a continuous real valued function on $X$. Given $\varepsilon>0$, then for every positive integer $n$, there exists an open cover $\alpha_{n}$ of $X$ such that $\operatorname{diam}(f U) \leq \varepsilon / n$ for all $U$ in $\alpha_{n}$ and

$$
\lim _{n \rightarrow \infty} \frac{1}{n} H\left(\alpha_{n}\right)=0 \text {. }
$$

Proof. Let $g=f+\alpha$ where $\alpha>\left|\inf _{x \in X}(f x)\right|$. Proposition 2.1 and the fact that $\operatorname{diam}(f U)=\operatorname{diam}(g U)$ for every subset $U$ of $X$ finish the proof.

Claim 2. Let $f X \rightarrow R^{m}$ be a continuous function from a metric space $X$ into $\left(R^{m}, d_{\infty}\right)$, where $d_{\infty}(X, Y)=\max \left\{\left|x_{i}-y_{i}\right|: i=\right.$ $1,2,3, \ldots, m\}$. Given $\varepsilon>0$, then for every positive integer $n$, there exists an open cover $\alpha_{n}$ of $X$ such that $\operatorname{diam}(f U) \leq \varepsilon / n$ for all $U$ in $\alpha_{n}$ and

$$
\lim _{n \rightarrow \infty} \frac{1}{n} H\left(\alpha_{n}\right)=0
$$

Proof. Let $\Pi_{i}: R^{n} \rightarrow R$ be the natural projection over $R$ (i.e., $\left.\Pi_{i}\left(x_{1}, x_{2}, \ldots, x_{m}\right)=x_{i}\right)$. For an integer $n>0$ let $\alpha_{n_{1}}$ be an open cover for $X$ satisfying Claim 2 with respect to $\Pi_{i} f$ for $i=1,2, \ldots, m$. Take 
$\alpha_{n}=\bigvee_{i=1}^{m} \alpha_{n_{i}}$. Then $\alpha_{n}$ is an open cover for $X$ and $\operatorname{diam}(f U) \leq \varepsilon / n$ for every $U \in \alpha_{n}$. Hence

$$
\lim _{n \rightarrow \infty} \frac{1}{n} H\left(\alpha_{n}\right) \leq \sum_{i=1}^{m} \lim _{n \rightarrow \infty} \frac{1}{n} H\left(\alpha_{n_{i}}\right)=0 .
$$

Claim 3. Let $f: X \rightarrow R^{m}$ be a continuous function from $X$ into $\left(R^{m}, d\right)$ where $d$ is a metric on $R^{m}$ which is $L$-equivalent to $d_{\infty}$. Given $\varepsilon>0$, then for every integer $n>0$, there exists an open cover $\alpha_{n}$ of $X$ such that $\operatorname{diam}(f U) \leq \varepsilon / n$ for all $U$ in $\alpha_{n}$ and

$$
\lim _{n \rightarrow \infty} \frac{1}{n} H\left(\alpha_{n}\right)=0 \text {. }
$$

Proof. Is an easy exercise for the reader.

From Claim 3 we obtain immediately:

THEOREM 1. Let $f: X \rightarrow Y$ be a continuous map from a metric space $X$ into a metric space $Y$ and suppose that $Y$ is L-embedded in the Euclidean space $R^{m}$ for some positive integer $m$. Given $\varepsilon>0$. Then for every integer $n>0$, there exists an open cover $\alpha_{n}$ of $X$ such that $\operatorname{diam}(f U) \leq \varepsilon / n$ for all $U \in \alpha_{n}$ and

$$
\lim _{n \rightarrow \infty} \frac{1}{n} H\left(\alpha_{n}\right)=0 \text {. }
$$

Corollary. Let $f: X \rightarrow X$ be a continuous map from $X$ into itself and suppose that $X$ is L-embedded in the Euclidean space $R^{m}$ for some $m>0$. Given $\varepsilon>0$, then for every integer $n>0$, there exists an open cover $\alpha_{n}$ of $X$ such that $\operatorname{diam}(U) \leq \varepsilon / n$ and $\operatorname{diam}(f U) \leq \varepsilon / n$ for all $U \in \alpha_{n}$ and

$$
\lim _{n \rightarrow \infty} \frac{1}{n} H\left(\alpha_{n}\right)=0 \text {. }
$$

Proof. If $f: X \rightarrow X$ is continuous and $X$ is $L$-embedded in $R^{m}$ for some $m>0$, then without loss of generality we can consider $f$ as a continuous function from $X$ into $R^{m}$. This can be done also for an identity map $I: X \rightarrow X$. Given $\varepsilon>0$ and a positive integer $n$, Theorem 1 implies that there exist open covers $\beta_{n}$ and $\gamma_{n}$ of $X$ such that $\operatorname{diam}(f U) \leq \varepsilon / n$ for every $U \in \beta_{n}$ and $\operatorname{diam}(W) \leq \varepsilon / n$ for every $W \in \gamma_{n}$ with

$$
\lim _{n \rightarrow \infty} \frac{1}{n} H\left(\beta_{n}\right)=0, \quad \lim _{n \rightarrow \infty} \frac{1}{n} H\left(\gamma_{n}\right)=0 .
$$


Let $\alpha_{n}=\beta_{n} \vee \gamma_{n}$. Then $\alpha_{n}$ is an open cover for $X$ with

$$
\max \{\operatorname{diam}(U), \operatorname{diam}(f U)\} \leq \varepsilon / n
$$

for every $U \in \alpha_{n}$ and

$$
\lim _{n \rightarrow \infty} \frac{1}{n} H\left(\alpha_{n}\right) \leq \lim _{n \rightarrow \infty} \frac{1}{n} H\left(\beta_{n}\right)+\lim _{n \rightarrow \infty} \frac{1}{n} H\left(\gamma_{n}\right)=0 .
$$

This finishes the proof.

3. Topological entropy and examples. Let $f: X \rightarrow X$ be continuous. For $E \subseteq X$ we say $E(n, \varepsilon)$-spans $X[\mathbf{1}, \mathbf{1 0}]$, if for each $x \in X$ there is an $e \in E$ so that $d\left(f^{i} x, f^{i} e\right) \leq \varepsilon$ for all $0 \leq i \leq n$. We let $r_{n}(X, \varepsilon)=$ $r_{n}(X, \varepsilon, f)$ denote the minimum cardinality of a set which $(n, \varepsilon)$-spans $X$. We define

$$
h(f, \varepsilon)=\lim _{n \rightarrow \infty} \sup \frac{1}{n} \log r_{n}(X, \varepsilon) .
$$

Notice that $h(f, \varepsilon)$ increases as $\varepsilon$ decreases. Finally, we define the topological entropy $h(f)$ by

$$
h(f)=\lim _{\varepsilon \rightarrow 0} h(f, \varepsilon) .
$$

In order to show that $L$-embedded condition is necessary in Theorem 1 we need to rewrite the corollary of Theorem 1 as follows:

Proposition 3.1. If $f: X \rightarrow X$ is continuous and the metric space $X$ is L-embedded in Euclidean space $R^{m}$ for some $m>0$, then

$$
\lim _{n \rightarrow \infty} \frac{1}{n} \log r_{1}(X, \varepsilon / n)=0 .
$$

Proof. By the corollary of Theorem 1 we let $\alpha_{n}$ be an open cover of $X$ with

$$
\max \{\operatorname{diam}(U), \operatorname{diam}(f U)\} \leq \varepsilon / n
$$

for every $U \in \alpha_{n}$ and $\lim _{n \rightarrow \infty} \frac{1}{n} H\left(\alpha_{n}\right)=0$. Pick a point in $U$ and let $E_{n}$ be the set of all such points. It is obvious that $E_{n}$ is $(1, \varepsilon / n)$ spanning set of $X$ and $\operatorname{card}\left(E_{n}\right) \leq \operatorname{card}\left(\alpha_{n}\right)$. This finishes the proof.

The following example [10] shows that $L$-embedded condition is necessary in Theorem 1.

EXAMPLE 3.2. Let $k$ be a fixed positive integer and let $C=\{0,1$, $2, \ldots, k-1\}$ with the discrete topology. Consider the product space $\Sigma=\prod_{-\infty}^{\infty} C$ with the product topology and the shift homeomorphism $\sigma: \Sigma \rightarrow \Sigma$ defined by $\sigma\left(\left\{w_{n}\right\}_{-\infty}^{\infty}\right)=\left\{w_{n+1}\right\}_{-\infty}^{\infty}$. A metric on $\Sigma$ can be 
defined by $d\left(\left\{x_{i}\right\},\left\{y_{i}\right\}\right)=1 /(m+2)$ if $m$ is the largest positive integer with $x_{i}=y_{i}$ for all $|i| \leq m$ and $d\left(\left\{x_{i}\right\},\left\{y_{i}\right\}\right)=1$ if $x_{0} \neq y_{0}$. Now it is an easy exercise to show that $r_{1}(\Sigma, 1)=1$ and $r_{1}\left(\Sigma, \frac{1}{n}\right) \geq k^{n}$ for every positive integer $n$. This means that

$$
\lim _{n \rightarrow \infty} \frac{1}{n} \log r_{1}\left(\Sigma, \frac{1}{n}\right) \geq \log k>0
$$

which contradicts Proposition 3.1 and shows that $(\Sigma, d)$ is not $L$ embedded in any Euclidean space.

Now we consider the following example:

EXAMPLE 3.3. One considers Smale's horseshoe [7], i.e., a diffeomorphism $f: D \rightarrow D$, where $D$ is a 2-dimensional disk. We may assume that $f / \partial D$ is the identity map. For more details, see the book of Nitecki [4]. This example has the property that for every positive integer $n$, there is $\varepsilon>0$ such that

$$
h\left(f^{n}, \varepsilon\right)=n \log 2 .
$$

Now we consider a sequence of disks $D_{n}$ on the plane of radii $2^{-n}$, disjoint and converging to a point. Let us also fix a sequence of natural numbers $\left\{n_{i}\right\}_{i=1}^{\infty}$. We define a map $g: R^{2} \rightarrow R^{2}$ as follows:

$$
g(x)= \begin{cases}f_{i}^{n_{1}}(x), & \text { if } x \in D_{i}, \\ x, & \text { if } x \in R^{2} \backslash \bigcup_{i=1}^{\infty} D_{i} .\end{cases}
$$

Here $f_{i}: D_{i} \rightarrow D_{i}$ is a homothetic copy of $f$. Obviously, $g$ extends to the one-point compactification $S^{2}$ and we can say

$$
h\left(g, \varepsilon_{i}\right) \geq h\left(f_{i}^{n_{l}}, \varepsilon_{i}\right) \geq n_{i} \log 2 .
$$

Here also $\varepsilon_{i}$ is a homothetic copy of $\varepsilon$.

The question we want to discuss here is whether it is possible to choose a sequence of natural numbers $\left\{n_{i}\right\}_{i=1}^{\infty}$ and a sequence of $\left\{\varepsilon_{i}\right\}_{i=1}^{\infty}$ such that $\varepsilon_{i} \cdot n_{i} \rightarrow \infty$ (i.e., is it possible to construct a $g: S^{2} \rightarrow S^{2}$ with the property that $\varepsilon_{i} h\left(g, \varepsilon_{i}\right) \rightarrow \infty$ as $\left.i \rightarrow \infty\right)$. Note that $\varepsilon_{i}$ is not independent of $n_{i}$; otherwise such a question is trivially true. In fact the answer for this question is not true. Moreover, we show later in Theorem 2 that $\varepsilon_{i} h\left(g, \varepsilon_{i}\right)$ must always vanish (i.e., $\varepsilon_{i} h\left(g, \varepsilon_{i}\right) \rightarrow 0$ as $i \rightarrow \infty)$.

LEMMA 3.4. If $E$ is $(1, \varepsilon)$-spanning set of a metric space $X$, then for every positive integer $k$, there exists a set $W$ which is $(k, 2 \varepsilon)$-spanning set of $X$ and $\operatorname{card}(W) \leq(\operatorname{card}(E))^{k}$. 
Proof. Special case of Lemma 2.1 in [1].

THeorem 2. If $f: X \rightarrow X$ is a homeomorphism on $X$ and if $X$ is $L$-embedded in $R^{n}$ for some positive integer $n$, then $\varepsilon h(f, \varepsilon) \rightarrow 0$ as $\varepsilon \rightarrow 0$.

Proof. Let $E_{n}$ be any $(1, \varepsilon / 2 n)$-spanning set of $X$ with minimum cardinality. Using Lemma 3.4 there exists a set $W_{n}$ which $(p, \varepsilon / n)$ spans $X$ and $\operatorname{card}\left(W_{n}\right) \leq\left(\operatorname{card}\left(E_{n}\right)\right)^{p}$ for every positive integer $p$. Hence

Therefore

$$
\frac{1}{p} \log r_{p}(X, \varepsilon / n) \leq \log r_{1}(X, \varepsilon / 2 n) .
$$

$$
h(f, \varepsilon / n) \leq \log r_{1}(X, \varepsilon / 2 n) .
$$

This means that

$$
\lim _{n \rightarrow \infty} \frac{1}{n} h(f, \varepsilon / n) \leq \lim _{n \rightarrow \infty} \frac{1}{n} \log r_{1}(X, \varepsilon / 2 n) .
$$

Proposition 3.1 finishes the proof of this theorem.

4. Topological entropy of expansive maps. During the remainder of this section we assume that $f$ is an expansive homeomorphism of a compact metric space $(X, d)$ onto itself with expansive constant $e>0$ (i.e., $x \neq y$ implies $d\left(f^{n} x, f^{n} y\right) \geq e$ for some integer $n$ ).

In this section we will use an adaptation of work by Reddy [6] to show that we can find a metric compatible with the topology of $X$ and a positive real number $\lambda, 0<\lambda<1$, such that $\lim _{m \rightarrow \infty} \frac{1}{m} \log r_{0}\left(X, \lambda^{m}\right)$ is the topological entropy of $f$ (i.e., the present tells us about the past and the future).

For an integer $n \geq 0$ define,

$$
W_{n}=\left\{(x, y) \in X \times X: d\left(f^{i} x, f^{i} y\right) \leq e \text { for }-n \leq i \leq n\right\} .
$$

It is obvious that $\cap W_{n}=\Delta$ where $\Delta=\{(x, x): x \in X\}$.

LEMMA 4.1. $\forall \varepsilon>0, \exists N>0$, such that $d\left(f^{i} x, f^{i} y\right) \leq e$ for all $i$ with $|i| \leq N$, implies $d(x, y) \leq \varepsilon$.

\section{Proof. Walters [11].}

Take $\varepsilon$ small enough such that $3 \varepsilon \leq e$. Choose $N$ using the above lemma with respect to $\varepsilon$. Define $V_{n}=W_{n N}$ for $n=0,1,2,3, \ldots$

The following was proved by W. Reddy [6]. Because $V_{n}$ is defined slightly differently in [6] we will give another proof. 
LEMMA 4.2 (cf. [6, Lemma 2]. The sequence $\left\{V_{n}\right\}$ is a nested sequence of symmetric neighborhoods of $\Delta$ whose intersection is $\Delta$ and such that $V_{n+1} \circ V_{n+1} \circ V_{n+1} \subseteq V_{n}$ for each $n \geq 0$.

Proof. Let $(x, y) \in V_{n+1} \circ V_{n+1} \circ V_{n+1}$. There exist $a, b$ elements in $X$ such that $(x, a) \in V_{n+1},(a, b) \in V_{n+1}$, and $(b, y) \in V_{n+1}$. Hence

$$
\begin{array}{ll}
d\left(f^{i} x, f^{i} a\right) \leq e & \text { for }-(n+1) N \leq i \leq(n+1) N \\
d\left(f^{i} a, f^{i} b\right) \leq e & \text { for }-(n+1) N \leq i \leq(n+1) N
\end{array}
$$

and

$$
d\left(f^{i} b, f^{i} y\right) \leq e \text { for }-(n+1) N \leq i \leq(n+1) N .
$$

Lemma 4.1 implies that

$$
\begin{array}{ll}
d\left(f^{i} x, f^{i} a\right) \leq \varepsilon & \text { for }-n N \leq i \leq n N, \\
d\left(f^{i} a, f^{i} b\right) \leq \varepsilon & \text { for }-n N \leq i \leq n N,
\end{array}
$$

and

$$
d\left(f^{i} b, f^{i} y\right) \leq \varepsilon \text { for }-n N \leq i \leq n N .
$$

The triangle inequality implies

$$
d\left(f^{i} x, f^{i} y\right) \leq 3 \varepsilon \leq e \text { for }-n N \leq i \leq n N .
$$

This means that $(x, y) \in V_{n}$.

The following is an immediate consequence of Lemma 4.2 and the Metrization lemma [3].

LEMMA 4.3. There is a metric $\rho$ compatible with the topology of $X$ such that

$$
N\left(\Delta ; 1 / 2^{n+1}\right) \subseteq V_{n} \subseteq N\left(\Delta ; 1 / 2^{n}\right)
$$

for $n \geq 1$.

LEMMA 4.4. There is a metric $\rho$ compatible with the topology of $X$ and there is $\lambda, 0<\lambda<1$, such that

$$
N\left(\Delta ; \lambda^{m+2 N}\right) \subseteq W_{m} \subseteq N\left(\Delta ; \lambda^{m-N}\right)
$$

for all $m \geq 0$.

Proof. Suppose $m=n N+j$ where $0 \leq j<N$. It is obvious that

$$
V_{n+1}=W_{(n+1) N}=W_{n N+N} \subseteq W_{n N+j}=W_{m} \subseteq W_{n N}=V_{n} .
$$


Therefore $V_{n+1} \subseteq W_{m} \subseteq V_{n}$. Using Lemma 4.3 we have $N\left(\Delta ; 1 / 2^{n+2}\right)$ $\subseteq V_{n+1}$ and $V_{n} \subseteq N\left(\Delta ; 1 / 2^{n}\right)$. Take $\lambda=(1 / 2)^{1 / N}$. It is clear that

$$
\begin{aligned}
N\left(\Delta ; \lambda^{m+2 N}\right) & \subseteq N\left(\Delta ; \lambda^{m+2 N-j}\right)=N\left(\Delta ; \lambda^{n N+2 N}\right) \\
& =N\left(\Delta ;(1 / 2)^{n+2}\right) \subseteq V_{n+1} \subseteq W_{m} \subseteq V_{n} \\
& \subseteq N\left(\Delta ; 1 / 2^{n}\right)=N\left(\Delta ;(1 / 2)^{n N / N}\right) \\
& =N\left(\Delta ; \lambda^{n N}\right)=N\left(\Delta ; \lambda^{m-j}\right) \subseteq N\left(\Delta ; \lambda^{m-N}\right) .
\end{aligned}
$$

This finishes the proof of this lemma.

Lemma 4.5 (cf. [1, Theorem 2.4 and Corollary 2.5]). $\exists \varepsilon>0$ such that $h(f)=h(f, \varepsilon)$ and $\frac{1}{n} \log r_{n}(X, \varepsilon) \rightarrow h(f)$.

THEOREM 3. There is a metric $\rho$ compatible with the topology of $X$ and there is $\lambda, 0<\lambda<1$, such that

$$
h(\phi)=\lim _{m \rightarrow \infty} \frac{1}{m} \log r_{0}\left(X, \lambda^{m}\right) .
$$

Proof. Let $E$ be $(2 m, e)$-spanning set of $X$ with minimum cardinality. It is obvious that for every $x \in X$, there exists $w \in f^{m}(E)$ so that $d\left(f^{i} x, f^{i} w\right) \leq e$ for $-m \leq i \leq m$. So $(x, w) \in W_{m}$. Using Lemma 4.4 we can find a metric $\rho$ on $X$ and $\beta, 0<\beta<1$, such that $(x, w) \in N\left(\Delta ; \beta^{m-N}\right)$. This means that there exists an $F$ which is $\left(0, \beta^{m-N}\right)$-spanning set of $X$ (i.e., $\beta^{m-N}$-net with respect to $\rho$ ) and $\operatorname{card}(F) \leq \operatorname{card}\left(f^{m} E\right)=\operatorname{card}(E)$. Therefore $r_{0}\left(X, \beta^{m-N}\right) \leq r_{2 m}(X, e)$.

Now suppose $F$ is $\left(0, \beta^{m+2 N}\right)$-spanning set of $X$ with respect to the metric $\rho$ and with minimum cardinality. Thus for every $x \in X$, there exists $w \in F$ such that $(x, w) \in N\left(\Delta ; \beta^{m+2 N}\right)$. So $(x, w) \in$ $W_{m}$ (i.e., $d\left(f^{i} x, f^{i} w\right) \leq e$ for $\left.-m \leq i \leq m\right)$. This implies that $d\left(f^{i} f^{-m} x, f^{i} f^{-m} w\right) \leq e$ for all $0 \leq i \leq 2 m$. Thus we can find $E$ which is $(2 m, e)$-spanning set of $X$ and $\operatorname{card}(E) \leq \operatorname{card}(F)$. Therefore $r_{2 m}(X, e) \leq r_{0}\left(X, \beta^{m+2 N}\right)$. Take $\lambda=\beta^{1 / 2}$. Therefore $r_{0}\left(X, \lambda^{2 m-2 N}\right) \leq$ $r_{2 m}(X, e)$ and $r_{2 m}(X, e) \leq r_{0}\left(X, \lambda^{2 m+4 N}\right)$. Using Lemma 4.5 and taking $e \leq \varepsilon$ we have

$$
h(\phi)=\lim _{m \rightarrow \infty} \frac{1}{2 m} \log r_{0}\left(X, \lambda^{2 m}\right) .
$$

But

$$
\frac{1}{2 m+1} \log r_{0}\left(X, \lambda^{2 m+1}\right) \geq \frac{1}{2 m+1} \log r_{0}\left(X, \lambda^{2 m}\right) \rightarrow h(\phi),
$$

and

$$
\frac{1}{2 m-1} \log r_{0}\left(X, \lambda^{2 m-1}\right) \leq \frac{1}{2 m-1} \log r_{0}\left(X, \lambda^{2 m}\right) \rightarrow h(\phi) .
$$




\section{Therefore}

$$
h(\phi)=\lim _{m \rightarrow \infty} \frac{1}{m} \log r_{0}\left(X, \lambda^{m}\right)
$$

and the proof is finished.

\section{REFERENCES}

[1] R. Bowen, Entropy-expansive maps, Trans. Amer. Math. Soc., 164 (1972), 323331.

[2] R. Bowen and P. Walters, Expansive one-parameter flows, J. Differential Equations, 12 (1972), 180-193.

[3] J. L. Kelley, General Topology, Van Nostrand, Princeton, NJ, 1955.

[4] Z. Nitecki, Differentiable Dynamics: An Introduction to the Orbit Structure of Diffeomorphisms. M. I. T. Press: Cambridge, 1971.

[5] T. Ohno, A weak equivalence and topological entropy, J. Kyoto Univ., 18 (1980), 289-298.

[6] W. Reddy, Expansive canonical coordinates are hyperbolic, Topology Appl., 15 (1983), 205-210.

[7] S. Smale, Differentiable dynamical systems, Bull. Amer. Math. Soc., 73 (1967), 747-817.

[8] R. Thomas, Topological stability: some fundamental properties, J. Differential Equations, 59 (1985), 103-122.

[9] _ Entropy of expansive flows, Ergodic Theory and Dynamical Systems, to appear.

[10] P. Walters, Ergodic Theory, Springer Lecture Notes, Vol. 458 (1975).

[11] _- On the Pseudo Orbit Tracing Property and its Relation to Stability, Lecture Notes in Mathematics, Vol. 668, pp. 231-244, Springer-Verlag, Berlin, 1977.

Received December 7, 1987.

EMbry-Riddle Aeronautical University

PrescotT, AZ 86301 


\section{PACIFIC JOURNAL OF MATHEMATICS EDITORS}

\author{
V. S. VARADARAJAN \\ (Managing Editor) \\ University of California \\ Los Angeles, CA 90024-1555-05 \\ Herbert Clemens \\ University of Utah \\ Salt Lake City, UT 84112 \\ THOMAs ENRIGHT \\ University of California, San Diego \\ La Jolla, CA 92093
}

R. FINN

Stanford University

Stanford, CA 94305

HermanN FlaschKa

University of Arizona

Tucson, AZ 85721

VAughan F. R. Jones

University of California

Berkeley, CA 94720

STEVEN KeRCKHOFF

Stanford University

Stanford, CA 94305

\author{
RobION KIRBY \\ University of California \\ Berkeley, CA 94720 \\ C. C. MoOre \\ University of California \\ Berkeley, CA 94720
}

Harold Stark

University of California, San Diego

La Jolla, CA 92093

\section{ASSOCIATE EDITORS
R. ARENS
E. F. BECKENBACH
B. H. NeumanN
F. WoLF
K. YoshidA (1906-1982)}

\section{SUPPORTING INSTITUTIONS}

UNIVERSITY OF ARIZONA
UNIVERSITY OF BRITISH COLUMBIA
CALIFORNIA INSTITUTE OF TECHNOLOGY
UNIVERSITY OF CALIFORNIA
MONTANA STATE UNIVERSITY
UNIVERSITY OF NEVADA, RENO
NEW MEXICO STATE UNIVERSITY
OREGON STATE UNIVERSITY

\author{
UNIVERSITY OF OREGON \\ UNIVERSITY OF SOUTHERN CALIFORNIA \\ STANFORD UNIVERSITY \\ UNIVERSITY OF HAWAII \\ UNIVERSITY OF TOKYO \\ UNIVERSITY OF UTAH \\ WASHINGTON STATE UNIVERSITY \\ UNIVERSITY OF WASHINGTON
}

The Supporting Institutions listed above contribute to the cost of publication of this Journal, but they are not owners or publishers and have no responsibility for its content or policies.

Mathematical papers intended for publication in the Pacific Journal of Mathematics should be in typed form or offset-reproduced (not dittoed), double spaced with large margins. Please do not use built up fractions in the text of the manuscript. However, you may use them in the displayed equations. Underline Greek letters in red, German in green, and script in blue. The first paragraph must be capable of being used separately as a synopsis of the entire paper. In particular it should contain no bibliographic references. Please propose a heading for the odd numbered pages of less than 35 characters. Manuscripts, in triplicate, may be sent to any one of the editors. Please classify according to the scheme of Math. Reviews, Index to Vol. 39. Supply name and address of author to whom proofs should be sent. All other communications should be addressed to the managing editor, or Elaine Barth, University of California, Los Angeles, California 90024-1555-05.

There are page-charges associated with articles appearing in the Pacific Journal of Mathematics. These charges are expected to be paid by the author's University, Government Agency or Company. If the author or authors do not have access to such Institutional support these charges are waived. Single authors will receive 50 free reprints; joint authors will receive a total of 100 free reprints. Additional copies may be obtained at cost in multiples of 50 .

The Pacific Journal of Mathematics is issued monthly as of January 1966. Regular subscription rate: $\$ 190.00$ a year (5 Vols., 10 issues). Special rate: $\$ 95.00$ a year to individual members of supporting institutions.

Subscriptions, orders for numbers issued in the last three calendar years, and changes of address should be sent to Pacific Journal of Mathematics, P.O. Box 969, Carmel Valley, CA 93924, U.S.A. Old back numbers obtainable from Kraus Periodicals Co., Route 100, Millwood, NY 10546.

The Pacific Journal of Mathematics at P.O. Box 969, Carmel Valley, CA 93924 (ISSN 0030-8730) publishes 5 volumes per year. Application to mail at Second-class postage rates is pending at Carmel Valley, California, and additional mailing offices. Postmaster: send address changes to Pacific Journal of Mathematics, P.O. Box 969, Carmel Valley, CA 93924.

\section{PUBLISHED BY PACIFIC JOURNAL OF MATHEMATICS, A NON-PROFIT CORPORATION}

Copyright (C) 1990 by Pacific Journal of Mathematics 


\section{Pacific Journal of Mathematics}

\section{Vol. 141, No. 2 December, 1990}

Ulrich F. Albrecht, Locally $A$-projective abelian groups and



Michel Carpentier, Sommes exponentielles dont la géométrie est très belle: $p$-adic estimates ..................................... 229

G. Deferrari, Angel Rafael Larotonda and Ignacio Zalduendo, Sheaves and functional calculus

Jane M. Hawkins, Properties of ergodic flows associated to product odometers ........................................287

Anthony To-Ming Lau and Viktor Losert, Complementation of certain subspaces of $L_{\infty}(G)$ of a locally compact group ............... 295

Shahn Majid, Matched pairs of Lie groups associated to solutions of the

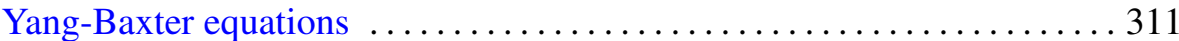

Diego Mejia and C. David (Carl) Minda, Hyperbolic geometry in $k$-convex



Vladimír Müller, Kaplansky's theorem and Banach PI-algebras ...........355

Raimo Näkki, Conformal cluster sets and boundary cluster sets coincide . . . 363

Tomasz Przebinda, The wave front set and the asymptotic support for $p$-adic groups

R. F. Thomas, Some fundamental properties of continuous functions and topological entropy 